\title{
PRIME ENTIRE FUNCTIONS
}

\author{
BY \\ FRED GROSS( $\left.{ }^{1}\right)$
}

\begin{abstract}
Factorizations of various functions are discussed. Complete factorizations of certain classes of functions are given. In particular it is shown that there exist primes of arbitrary growth.
\end{abstract}

I. Introduction. A meromorphic function $h(z)=f(g(z))$ is said to have $f(z)$ and $g(z)$ as left and right factors respectively, provided that $f(z)$ is meromorphic and $g(z)$ is entire ( $g$ may be meromorphic when $f(z)$ is rational). $h(z)$ is said to be $E$ prime ( $E$-pseudo prime) if every factorization of the above form into entire factors implies that one of the functions $f(z)$ or $g(z)$ is linear (a polynomial). $h(z)$ is said to be prime (pseudo prime) if every factorization of the above form, where the factors may be meromorphic, implies that one of $f(z)$ or $g(z)$ is linear (a polynomial or $f(z)$ is rational).

[1]-[11], [14] and [17] have dealt with various factorization problems. In particular, the following result on primes was proved in [1].

THEOREM 1. Let $H(z)$ be a periodic entire function of finite lower order and let a be a nonzero constant. Then $H(z)+a z$ is prime.

This result generalized an earlier assertion of Rosenbloom [17] proved in [7], that $e^{z}+z$ is prime. The more difficult problem of whether $\exp \left(e^{z}\right)+z$ is prime was left open.

In this paper we shall weaken considerably the condition that $H$ is of finite lower order. In a sense we shall show that our growth condition on $H$ is about the best that can be assumed. However, we then proceed to show that when $H$ is of the form $e^{\alpha}, \alpha$ entire, one can say much more about $H(z)+a z$. In particular, it will follow that $\exp \left(e^{z}\right)+z$ is prime. The question, however, whether $e_{n}(z)+z$ is prime for $n>2$ remains open. Here $e_{n}(z)$ denotes the $n$th iterate of the exponential function. We next study the primes of the forms (1) $Q(z) e^{H(z)+S(z)}$ where $Q(z)$ ( $\not \equiv$ constant) and $S(z)$ are polynomials and $H(z)$ is entire and periodic such that $H(z)+S(z)$ is not a constant. This problem is solved completely; in fact all possible factorizations of the function of the form (1) are found. This class of primes gives us primes of arbitrarily large growth. We shall in fact exhibit primes of arbitrary

Received by the editors June 19, 1970 and, in revised form, March 9, 1971.

AMS 1969 subject classifications. Primary 3060.

Key words and phrases. Primes, factors, factorization, meromorphic functions.

(1) This research was partially supported by the National Science Foundation under grant number GP-13875. 
growth including primes of prescribed order and type. Among the primes of arbitrarily large growth that are exhibited are a class of almost periodic entire functions. These are of particular interest, since there are no known periodic entire primes. Periodic $E$-primes, however, do exist [11].

We shall assume in the sequel that the reader is familiar with Nevanlinna theory and the functions $T(r, f), N(r, f), m(r, f)$, etc.

II. Primes of the form $H(z)+a z$. Let $M_{F}(r)$ denote the maximum modulus function of $F$.

In this section we prove

THEOREM 2. Let $H(z)$ be periodic and entire and let a be any nonzero constant. If for every positive $\varepsilon$, there exist an infinite sequence of $r$ approaching infinity such that

$$
M_{H}(r)<e_{2}(\varepsilon r)
$$

then $F(z)=H(z)+a z$ is prime.

We begin with some lemmas essential to the proof.

Lemma 1 (Polya [15]). Let $f$ and $g$ be entire. For any $\delta, 0<\delta<1$, there exists an appropriate positive constant $c$ such that

$$
M_{f(g)}(r)>M_{f}\left(c M_{g}(\delta r)\right)
$$

for all sufficiently large $r$, where $M_{F}(r)$ denotes the maximum modulus function of $F$.

LEMMA 2. Let $d$ and $\tau$ be two numbers such that $d \tau / 2 \pi i$ is an integer. Let $T(z)(\not \equiv 0)$ be a polynomial of degree $t$ and let $H(z)$ and $\theta(z)$ be periodic and entire with period $\tau$. For any factorization of

$$
F(z)=H(z)+T(z) e^{\theta(z)+d z}
$$

into entire factors, the right factor $g(z)$ must be of the form

$$
g(z)=H_{1}(z)+S(z) \exp \left(H_{2}(z)+c z\right)
$$

where $H_{i}(z)$ are periodic and entire with period $\tau, c$ is a constant and $S(z)$ is a polynomial of degree $s$. Furthermore, $s \leqq t$ if $e^{c \tau}=1$ and $s \leqq t-1$ otherwise.

Proof. See proof of Theorem 1 in [1].

LEMMA 3. If $T(z)$ is nonconstant and of first degree, then $c$ in Lemma 2 satisfies $e^{c \tau}=1$.

Proof. Assume $e^{c \tau} \neq 1$. Then for some entire left factor $f(w),(2)$ yields for every integer $n$

$$
f\left(H_{1}(z)+K \exp \left(H_{2}(z)+c z\right) \cdot e^{c n \tau}\right)=H(z)+T(z+n \tau) e^{\theta(z)}
$$


where $K$ is a constant. We may assume without any loss of generality that $\left|e^{c \tau}\right| \leqq 1$. Thus, for a fixed $z$, the left side of (4) is bounded in $n$ while the right side is not, a contradiction. Hence, our assertion follows.

Lemma 4 [11]. Let $F(z)$ be nonperiodic and entire. If $F(z)$ is E-prime, then it is prime.

LEMMA 5. If $h(z)$ is meromorphic (and $\not \equiv 0$ ) and for all positive integers $n, h(n)=0$, then the lower order (denoted by $\lambda(h)$ ) satisfies $\lambda(h) \geqq 1$. When $\lambda(h)=1$, the lower type (defined as $\lim \inf T(r, h) / r$ and denoted by $\tau(h)$ ) satisfies $\tau(h)>0$.

Proof.

$$
\begin{aligned}
& \lambda(h) \geqq \liminf _{r \rightarrow \infty} \log T(r, h) / \log r=\liminf _{r \rightarrow \infty} \log T(r, 1 / k) / \log r \\
& \geqq \liminf _{r \rightarrow \infty} \log N(r, 1 / h) / \log r \geqq 1-\varepsilon
\end{aligned}
$$

for any given positive $\varepsilon$ and sufficiently large $r$. A similar argument proves the assertion for $\tau(h)$.

LEMMA 6 [13]. Let $f_{i}(z)(i=1,2, \ldots, n)$ be meromorphic functions which satisfy for some constants $C_{i}$

$$
\sum_{i=1}^{n} C_{i} f_{i}=0
$$

If in addition the functions $f_{i}$ satisfy the following conditions:

(a) The ratios $f_{i} \mid f_{j}(i \neq j)$ are nonrational.

(b) If $T^{*}(r)$ is the minimum of $T\left(r, f_{i} / f_{j}\right), i \neq j$, and if $N\left(r, f_{i}\right)=\varepsilon_{i}(r) T^{*}(r), N\left(r, 1 / f_{i}\right)$ $=\eta_{i}(r) T^{*}(r)$, then there is a set $S$ of infinite measure on which $\max _{i, j}\left\{\varepsilon_{i}(r), \eta_{j}(r)\right\} \rightarrow 0$ as $r \rightarrow \infty$.

REMARK. The statement in [13] is somewhat weaker, but the proof there establishes the above assertion.

Proof of Theorem 2. We may assume without any loss of generality that $a=1$ and $H$ has period 1 . Suppose that

$$
F(z)=f(g(z))
$$

where $f$ and $g$ are entire. By Lemmas 2 and 3, for every integer $n$ and some constant $k(k \neq 0$ since $F$ is not periodic):

$$
g(z+n)-g(z)=k n \exp \left(H_{2}(z)+c z\right) .
$$

Fix $z=z_{0}$ and put $k \exp \left(H_{2}\left(z_{0}\right)+c z_{0}\right)=\lambda, h(z)=g\left(z_{0}+z\right)-\lambda z-g\left(z_{0}\right)$. Then $h(n)=0$ for each $n$ and Lemma 5 shows that either $h=0$ or $\lambda(h) \geqq 1$ or $\lambda(h)=1, \tau(h)>0$. Hence either $g$ is linear or $\lambda(g) \geqq 1$ or $\lambda(g)=1, \tau(g)>0$. 
Furthermore, by Lemma 3 we have

$$
f\left(H_{1}(z)+[k(z+n)+b] \exp \left(H_{2}(z)+c z\right)\right)=H(z)+z+n .
$$

Again fixing $z$ and applying Lemma 5, we conclude that $\lambda(f) \geqq 1$, and when $\lambda(f)=1$, $\tau(f)>0$. Applying Lemma 1 we arrive at the contradiction that for some $\varepsilon>0$

$$
M_{F}(r)>M_{f g}(r)>e_{2}(\varepsilon r)
$$

for sufficiently large $r$. The theorem follows immediately by Lemma 4 .

The function

$$
F(z)=\exp (\exp (z)+z)+\exp z+z=G(G(z)),
$$

where $G(z)=\exp (z)+z$, illustrates that the growth condition in Theorem 2 is fairly sharp. Nevertheless we have

THEOREM 3. Let a be a nonzero constant and $H(z)$ be periodic, entire and satisfy for any given $\varepsilon>0$ and some real $t$,

$$
M_{H}(r)<e_{2}((t+\varepsilon) r)
$$

for sufficiently large $r$. If $F(z)=H(z)+a z=f(g(z))$, where $f$ and $g$ are entire and nonlinear, then $g(z)$ must be of the form

$$
g(z)=\sum_{j=-m}^{m} \lambda_{j} \exp (2 \pi j i z / \tau)+B z e^{c z}
$$

where $2 \pi m /|\tau| \leqq t, c=2 \pi N i / \tau$ for some integer $N,|c| \leqq t, \tau$ is a period of $H$, and $\lambda_{j}$, $B$ are constants.

Proof. We may assume again that $\tau=1$ and $a=1$. Arguing as in the proof of the previous theorem, one easily verifies that $g(z)$ has the form

$$
g(z)=H_{1}(z)+z \exp \left(H_{2}(z)+c z\right), \quad\left(e^{c}=1\right)
$$

and that $f(z)$ is of lower order at least 1 .

If $H_{2}(z)$ is nonconstant, then by Lemma 5 , it is at least of lower order 1 . It then follows by Lemma 1 that for any $\varepsilon>0$

$$
M_{F}(r)>e_{3}((1-\varepsilon) r)
$$

for sufficiently large $r$. This contradicts our hypotheses and it follows that $H_{2}(z)$ must be a constant. Thus, we have

$$
g(z)=H_{1}(z)+B z e^{c z}
$$

for some constant $B$. By Lemma $1, g$ and hence $H_{1}$ are of exponential type and when $H_{1}$ is of order 1 , it is of type at most $t$. It follows that $g(z)$ must have the form (7).

Using Theorem 3, we prove 
THEOREM 4. Let $H(z)$ be periodic of period $\tau$, entire and of exponential type and let $s$ be any integer; then for any constant $a(\neq 0)$ the function

$$
F(z)=e^{H(z)+(2 \pi i s / \tau) z}+a z
$$

is prime.

Proof. We may assume that $a=1$ and $\tau=1$. Suppose that $F(z)=f(g(z))$, where $f$ and $g$ are entire. From Theorem 3

$$
g(z)=\sum_{j=-t}^{t} \lambda_{j} \exp (2 \pi i j z)+B z e^{c z} .
$$

Observe that in any strip $S,-\infty<x<\infty, a \leqq y \leqq b, F(z)=z+K(z)$, where $K(z)$ is bounded. In particular $|F(x)| \rightarrow \infty$ and hence $|g(x)| \rightarrow \infty$ as $x \rightarrow+\infty$ or $x \rightarrow-\infty$. This shows that in (10) $B \neq 0, \operatorname{Re} c=0$. If $c \neq 0$ then $u=B z e^{c z}$ maps $S$ onto a region covering $|u|>A$ for some $A$. Thus, in $S, F=f(g)$ takes all large values, which cannot be the case if $F(z)-z$ is bounded in $S$. Thus, $c=0$.

Since $f(g(z))$ has no fix-points (i.e. $f(g(z))=z$ has no solutions) neither does $g(f(z))$. Indeed, if for some $z_{0}, g\left(f\left(z_{0}\right)\right)=z_{0}$, then $f g\left(f\left(z_{0}\right)\right)=f\left(z_{0}\right)$. Thus it follows from (10) that there exists an entire function $\alpha(z)$ such that

$$
\sum_{-t}^{t} \lambda_{j} \exp (2 \pi i j f)+B f=e^{\alpha(z)}+z
$$

Suppose $g$ is not linear. Then let the order of the rational function $R(x)=\sum_{-t}^{t} \lambda_{j} x^{j}$ be $k$. Then

$$
T\{r, R(\exp (2 \pi i f))\} \sim k T(r, \exp (2 \pi i f)) .
$$

From (11) it follows that $T\left(r, e^{\alpha(z)}\right) \sim k T(r, \exp 2 \pi i f)$ as $r \rightarrow \infty$. Thus, the conditions of Lemma 6 apply to the functions $\exp (2 \pi i j f),-t \leqq j \leqq t, j \neq 0, \exp \alpha(z), B f-z+\lambda_{0}$, and we have a contradiction unless for every set $S$ of infinite measure there is an $\varepsilon>0$ such that for certain arbitrarily large $r$ in $S$

$$
T(r, f) \sim T(r, B f-z)>N(r, 1 /(B f-z))>\varepsilon T^{*}(r)
$$

where $T^{*}=T(r, \exp (\alpha-2 \pi i j f)$ for some $j$.

The set in which (12) holds must therefore have finite measure and we must have $|j|=k$.

Assume $j=k$ (the case $j=-k$ is treated similarly). Then

$$
\lambda_{k} e^{2 \pi i k f}+\lambda_{k-1} e^{2 \pi i(k-1) f}+\cdots+\lambda_{0}+B f-z=e^{\alpha(z)} .
$$

Divide through by $e^{2 \pi i k f}$ and we have

$$
\left\{\lambda_{k}-\exp (\alpha-2 \pi i k f)\right\}+\lambda_{k-1} \exp (-2 \pi i f)+\cdots+\left(\lambda_{0}+\beta f-z\right) \exp (-2 \pi i k f)=0 .
$$

Application of Lemma 6, noting that the characteristic of the first bracket is $O(T(r, f))$ on a set of infinite measure, gives a contradiction unless the coefficients of the exponentials are zero, so that $f$ is linear. 
COROLlary. For any $a \neq 0$, the function $\exp \left(e^{z}\right)+a z$ is prime.

III. Factorization of $Q(z) e^{H(z)+S(z)}$. We now prove

THEOREM 5. Let $Q(z)$ (not identically constant) and $S(z)$ be two polynomials. If $H(z)$ is periodic and entire, then any factorization with entire factors of any function of the form $F(z)=Q(z) e^{H(z)+S(z)}(H+S$ not identically constant $)$ has one of the forms

$$
F(z)=F(p(z))
$$

where $p(z)$ is a second degree polynomial, or

$$
F(z)=[g(z)]^{t},
$$

where $t$ is a positive integer.

In addition to some of the lemmas in §II, we shall need the following results.

Definition. An entire function $f$ is said to be periodic mod an entire function $g$ with period $\tau$ if $f(z+\tau)-f(z)=g(z)$.

LEMMA 7 [1]. Iff is nonconstant and entire and $p$ is a polynomial of degree greater than 2 , then $f(p)$ cannot be periodic mod any polynomial.

Lemma 8. Let $Q(z), S(z)$ and $H(z)$ be as in Theorem 5 and let $\tau$ be a period of $H$. If $\alpha$ is any nonconstant entire function, then $\alpha\left(Q(z) e^{H(z)+S(z)}\right)$ cannot be periodic mod any polynomial with period $\tau$.

Proof. Suppose that for some polynomial $T(z)$ we have

$$
\alpha\left(Q(z+\tau) e^{H(z)+S(z+\tau)}\right)-\alpha\left(Q(z) e^{H(z)+S(z)}\right)=T(z) .
$$

Choose $w_{0} \not \equiv 0$ and an infinite sequence $z_{i}(i=1,2, \ldots)$ approaching infinity such that $Q\left(z_{i}\right) \exp \left(H\left(z_{i}\right)+S\left(z_{i}\right)\right)=w_{0}$. We may write $Q(z+\tau)=Q(z)+Q_{0}(z)$ and $S(z+\tau)=S(z)+S_{0}(z)$, where degree $Q_{0}<$ degree $Q$ and degree $S_{0}<$ degree $S$ and $Q_{0} \not \equiv 0$. Thus, (16) becomes

$$
\alpha\left(\left[Q(z)+Q_{0}(z)\right] \exp \left(H(z)+S(z)+S_{0}(z)\right)\right)-\alpha\left(Q(z) e^{H(z)+S(z)}\right)=T(z)
$$

or

$$
\alpha\left(\left[w_{0}+\left(Q_{0}\left(z_{i}\right) / Q\left(z_{i}\right)\right) w_{0}\right] \exp \left(S_{0}\left(z_{i}\right)\right)\right)-\alpha\left(w_{0}\right)=T\left(z_{i}\right) .
$$

If $\operatorname{Re} S_{0}\left(z_{i}\right) \leqq 0(\operatorname{Re} A$ denotes real part of $A)$, then the arguments of $\alpha$ on the left side of the above equation have a limit point and $T$ and $\alpha$ must be constants, contrary to our hypotheses. We may therefore assume that for an infinite subsequence $\left\{z_{i}^{\prime}\right\}$ of $\left\{z_{i}\right\}$ approaching infinity, $\operatorname{Re} S_{0}\left(z_{i}^{\prime}\right)>0, i=1,2, \ldots$ Furthermore, $Q(z+\tau) e^{H(z)+S(z+\tau)}=w_{0}$ has the zeros $z_{i}^{\prime}-\tau$. Now (16) yields $\alpha\left(Q(z+\tau) e^{H(z)+S(z+\tau)}\right)$ $-\alpha\left(\left[Q(z+\tau)-Q_{0}(z)\right] \exp \left(H(z)+S(z+\tau)-S_{0}(z)\right)\right)=T(z)$. At $z=z_{i}^{\prime}-\tau$ we get

$$
\alpha\left(w_{0}\right)-\alpha\left(\left[w_{0}-\left(Q_{0}\left(z_{i}^{\prime}-\tau\right) / Q\left(z_{i}^{\prime}\right)\right) w_{0}\right] \exp \left(-S_{0}\left(z_{i}^{\prime}-\tau\right)\right)\right)=T\left(z_{i}^{\prime}\right) .
$$


Since $\operatorname{Re} S_{0}\left(z_{i}^{\prime}\right)>0$, it is clear that $\operatorname{Re}\left(-S_{0}\left(z_{i}^{\prime}-\tau\right)\right)<0$ for sufficiently large $i$. Hence, it follows again from the above equation that $\alpha$ must be constant, contrary to our hypotheses. This completes the proof of Lemma 8.

LEMMA 9 [12]. If $f(z)$ is a transcendental function and $a_{1}(z), a_{2}(z)$ are distinct meromorphic functions satisfying for $\nu=1$ and 2

$$
T\left(r, a_{v}(z)\right)=o(T(r, f)) \quad \text { as } r \rightarrow \infty,
$$

then

$$
\{1+o(1)\} T(r, f) \leqq \sum_{v=1}^{2} \bar{N}\left(r, 1 /\left(f-a_{v}(z)\right)\right)
$$

for all $r$ outside a set of finite measure.

We now proceed with the proof of the theorem.

Proof of Theorem 5. We first consider the case when $F=f(g)$, where $f$ is entire and $g$ is a polynomial. Clearly $f$ must have the form $f(w)=T(w) e^{\alpha(w)}$, where $T(w)$ is a polynomial $(\not \equiv 0)$ and $\alpha(w)$ is entire. Thus,

$$
f(g)=T(g) e^{\alpha(g)}=Q(z) e^{H(z)+S(z)} .
$$

Consequently, $\alpha(g)$ must be periodic modulo a polynomial. Hence, by Lemma 7, $g$ must be of degree 2 at most and this factorization reduces to the form (14).

Now suppose that $g$ is transcendental entire. Then $f(w)$ must have the form

$$
f(w)=(w-a)^{n} e^{\alpha(w)},
$$

where $a$ is a constant, $n$ a positive integer and $\alpha$ is an entire function. $g(z)$ must be of the form

$$
g(z)=Q_{1}(z) e^{\beta(z)}+a,
$$

where $Q_{1}$ is a polynomial which satisfies $Q_{1}^{n}=K Q$ for some constant $K$ and $\beta$ is nonconstant entire. Writing $\alpha^{*}(w)=\alpha(w+a)$ we obtain

$$
F=Q_{1}^{n} \exp \left(n \beta+\alpha^{*}\left(Q_{1} e^{\beta}\right)\right)=Q e^{H+S} .
$$

Thus,

$$
n \beta+\alpha^{*}\left(Q_{1} e^{\beta}\right)=H+S
$$

From (19) we get

$$
\alpha^{*}\left(Q_{1}(z+\tau) e^{\beta(z+\tau)}\right)-\alpha^{*}\left(Q_{1}(z) e^{\beta(z)}\right)=S(z+\tau)-S(z)-n \beta(z+\tau)+n \beta(z) .
$$

Equation (20) implies that either

(i) $\beta(z)=H_{1}(z)+(1 / n) S(z)$ and $\alpha^{*}\left(Q_{1}(z) e^{\beta(z)}\right)$ is periodic with period $\tau$, where $H_{1}(z)$ is entire and periodic with period $\tau$, or 
(ii) $Q_{1}(z+\tau) e^{\beta(z+\tau)}-Q_{1}(z) e^{\beta(z)}=\beta^{*}(z) e^{\gamma(z)}$, where $\beta^{*}$ and $\gamma$ are entire and

$$
T\left(r, \beta^{*}\right)=o\left(T\left(r, e^{\beta(z+\tau)-\beta(z)}\right)\right)
$$

unless $\beta(z+\tau)=\beta(z)$.

Applying Lemma 8 to case (i) and the case when $\beta(z+\tau)=\beta(z)$, we find that $\alpha^{*}$ and hence $\alpha$ must be constant so that the factorization reduces to the form (15). Assume, therefore that (21) holds. (ii) may be rewritten in the form

$$
Q_{1}(z+\tau) e^{\beta(z+\tau)-\beta(z)}-Q_{1}(z)=\beta^{*}(z) e^{\gamma(z)-\beta(z)}=U(z),
$$

say. It is clear from equation (22) that for some $A>0$ and sufficiently large $r$

$$
T\left(r, e^{\gamma-\beta}\right)>A T\left(r, e^{\beta(z+\tau)-\beta(z)}\right) .
$$

Also

$$
N\left(r, 1 /\left(U+Q_{1}\right)\right)=o(T(r, U)) \text { and } N(r, 1 / U)=o(T(r, U)) .
$$

Thus, by Lemma 9

$$
\{1+o(1)\} T(r, U) \leqq N\left(r, 1 /\left(U+Q_{1}\right)\right)+N(r, 1 / U)=o(T(r, U))
$$

for all $r$ outside a set of finite measure.

Equation (24) leads to a contradiction and our theorem follows.

COROllary 1. Let $Q(z)$ be a polynomial of degree 1 or a prime polynomial of degree greater than 2. If $H(z)$ is a periodic function and $S(z)$ is any polynomial such that $H$ and $S$ are not both constant, then $Q(z) e^{H(z)+S(z)}$ is prime.

Corollary 2. For $Q(z)$ as in Corollary 1 and any positive integer $n, Q e_{n}(z)$ is prime.

The primes we have exhibited are, to the author's knowledge, the first illustration of primes of arbitrarily large growth. In the next section we give examples of primes of arbitrarily large growth which are also almost periodic.

IV. Almost periodic primes. We start with a simple lemma.

LEMMA 10. If $f(z)$ is entire and maps the unit circle on the unit circle, then it must be of the form $f(z)=c z^{n}$ for some integer $n$ and some constant $c$ with $|c|=1$.

Proof. By the reflection principle a zero $z_{0}$ of $f(z)$ in $|z|<1$, corresponds to a pole $1 /\left(\bar{z}_{0}\right)=z_{1}$ of $f(z)$ in $|z|>1$. Since $f$ is entire, we see that the only zeros of $f(z)$ in $|z|<1$ lie at $z=0$ and if $z=0$ is an $n$-fold zero, then $f=c z^{n},|c|=1$.

The author is indebted to M. Newman for suggesting the proof of Lemma 10 .

We now prove

THEOREM 6. Let $H_{j}$ ( $\not \equiv$ constant) and $G_{j}$ be entire periodic functions with period $\tau_{j}$ and let $a_{j}$ be constants such that $a_{j} \tau_{j} / 2 \pi i$ is not rational $(j=1,2)$ and assume further 
that $\tau_{1} / \tau_{2}$ is irrational. Then the functions

$$
F_{j}(z)=H_{j}+\exp \left(a_{j} z+G_{j}(z)\right) \quad(j=1,2)
$$

when factored into entire factors have no common right factor.

Proof. Suppose that there are nonlinear entire functions $f_{j}$ and an entire function $g$ such that

$$
F_{j}(z)=f_{j}(g(z)) \quad(j=1,2) .
$$

Then (25) and (26) yield

$$
f_{j}\left(g\left(z+\tau_{j}\right)\right)-f_{j}(g(z))=\left(\exp \left(a_{j} \tau_{j}\right)-1\right) \exp \left(a_{j} z+G_{j}(z)\right) \quad(j=1,2) .
$$

We conclude by means of Lemma 2 as in our earlier arguments that

$$
g\left(z+\tau_{j}\right)-g(z)=\exp \left(\alpha_{j} z+I_{j}(z)\right)
$$

where $\alpha_{j}$ is constant and $I_{j}$ is periodic with period $\tau_{j}(j=1,2)$. From (27) and (28) we obtain

$$
\begin{aligned}
g\left(z+\tau_{1}+\tau_{2}\right)-g(z) & =\exp \left(\alpha_{2} \tau_{1}+I_{2}\left(z+\tau_{1}\right)+\alpha_{2} z\right)+\exp \left(\alpha_{1} z+I_{1}(z)\right) \\
& =\exp \left(\alpha_{1} \tau_{2}+I_{1}\left(z+\tau_{2}\right)+\alpha_{1} z\right)+\exp \left(\alpha_{2} z+I_{2}(z)\right) .
\end{aligned}
$$

Applying Lemma 6, one concludes that $I_{j}^{\prime}$ has periods $\tau_{1}$ and $\tau_{2}$ and therefore $I_{j}^{\prime}$ must be a constant $(j=1,2)$. It follows that $I_{j}$ must be a constant $c_{j}$, say $(j=1,2)$ since it is periodic. Consequently, one obtains from (29)

$$
\left(\exp \left(\alpha_{1} \tau_{2}\right)-1\right) \exp \left(\alpha_{1} z+c_{1}\right)=\left(\exp \left(\alpha_{2} \tau_{1}\right)-1\right) \exp \left(\alpha_{2} z+c_{2}\right) .
$$

Assume first that $\alpha_{1} \neq \alpha_{2}$. Then one gets from (30), $\alpha_{1} \tau_{2}=2 \pi n i, \alpha_{2} \tau_{1}=2 \pi m i$, where $n$ and $m$ are integers. Thus, from (28) we obtain as in earlier arguments that we used, that

$$
g(z)=J_{1}(z)+\lambda_{1} \exp \left(2 \pi n i z / \tau_{2}\right)=J_{2}(z)+\lambda_{2} \exp \left(2 \pi m i z / \tau_{1}\right),
$$

where $J_{j}$ is entire and periodic with period $\tau_{j}$ and $\lambda_{j}$ is a constant $(j=1,2)$. From (31) we see that

$$
J_{1}(z)-\lambda_{2} \exp \left(2 \pi m i z / \tau_{1}\right)=J_{2}(z)-\lambda_{1} \exp \left(2 \pi n i z / \tau_{2}\right)=G(z), \text { say. }
$$

$G(z)$ is thus doubly periodic and hence constant. We may therefore assume that $g(z)$ has the form

$$
g(z)=\lambda_{2} \exp \left(2 \pi m i z / \tau_{1}\right)+\lambda_{1} \exp \left(2 \pi n i z / \tau_{2}\right),
$$

where $\lambda_{1}, \lambda_{2} \neq 0$ since $F_{1}, F_{2}$ are not periodic.

Hence, by (25) and (32) we have

$$
f_{1}\left(\lambda_{2} \exp \left(2 \pi m i z / \tau_{1}\right)+\lambda_{1} \exp \left(2 \pi n i z / \tau_{2}\right)\right)=H_{1}(z)+\exp \left(\alpha_{1} z+G_{1}(z)\right)
$$


for any integer $k$, let $z=k \tau_{1}$. Then (33) becomes

$$
f_{1}\left(\lambda_{2}+\lambda_{1} \exp \left(2 \pi n i k \tau_{1} / \tau_{2}\right)\right)=H_{1}(0)+\exp \left(k a_{1} \tau_{1}+G_{1}(0)\right) .
$$

Since, $\tau_{1} / \tau_{2}$ is irrational, it follows from (34) that as $k$ runs through the positive integers, $\exp \left(k a_{1} \tau_{1}\right)$ has an infinity of limit points. The latter is only possible if either $a_{1} \tau_{1}$ is an irrational multiple of $\pi i$ or if $\left|\exp \left(a_{1} \tau_{1}\right)\right|<1$. A similar argument with the negative integers $k$ leads to the conclusion that either $a_{1} \tau_{1}$ is an irrational multiple of $\pi i$ or $\left|\exp \left(-a_{1} \tau_{1}\right)\right|<1$. Hence, in any case we must have $a_{1} \tau_{1}=s \pi i$, where $s$ is irrational. Thus, (34) implies that the entire function

$$
\exp \left(-G_{1}(0)\right)\left[f_{1}\left(\lambda_{2}+\lambda_{1} w\right)-H_{1}(0)\right]
$$

maps a dense set on the unit circle on a dense set on the unit circle. Thus, it must map the unit circle on the unit circle and, by Lemma $10, f_{1}$ must be a polynomial of the form

$$
f_{1}(w)=A(w-B)^{N}+C,
$$

where $A, B$ and $C$ are constants and $N$ is a positive integer. Using (35) and applying Lemma 6 to (33) one easily arrives at a contradiction unless $N=1$. Thus, we may assume that $\alpha_{1}=\alpha_{2}=\alpha$, say. From (30) we see that $\exp \left(\alpha \tau_{1}\right)=1$ implies $\exp \left(\alpha \tau_{2}\right)=1$, which is excluded, since $\tau_{1} / \tau_{2}$ is not rational. Then by (28)

$$
g(z)=J_{j}(z)+A_{j} e^{\alpha z}
$$

where $A_{j} \neq 0$ is constant, $j=1,2, J_{j}$ periodic with period $\tau_{j}$. Hence,

$$
J_{1}(z)+A_{1} e^{\alpha z}=J_{2}(z)+A_{2} e^{\alpha z}
$$

or

$$
A_{1} e^{\alpha z}\left(\exp \left(\alpha \tau_{1}\right)-1\right)=J_{2}\left(z+\tau_{1}\right)-J_{2}(z)+A_{2} e^{\alpha z}\left(\exp \left(\alpha \tau_{1}\right)-1\right),
$$

i.e., for a suitable constant $k, J_{2}\left(z+\tau_{1}\right)-J_{2}(z)=k e^{\alpha z}$. The left-hand side here is entire and of period $\tau_{2}$ and if $k \neq 0$ the right side is of period $2 \pi i / \alpha$ so the ratio $\tau_{2} \alpha / 2 \pi i$ is rational. Similarly $J_{1}\left(z+\tau_{2}\right)-J_{1}(z)=l e^{\alpha z}$ and, if $l \neq 0, \tau_{1} a / 2 \pi i$ is rational. Thus, if $k l \neq 0, \tau_{1} / \tau_{2}$ is rational. Hence, say $k=0$, then $J_{2}$ has period $\tau_{1}$ as well as $\tau_{2}$ and so $\tau_{1} / \tau_{2}$ is rational again, so $J_{2}$ is constant. It then follows that $J_{1}$ is constant. We may assume therefore, that the only common right factors are of the form $A e^{\alpha z}, A$ a constant. Hence, we may assume that

$$
H_{j}^{*}(z)=f_{j}\left(e^{\alpha z}\right)=H_{j}(z)+\exp \left(a_{j} z+G_{j}(z)\right) \quad(j=1,2) .
$$

Let $\tau_{3}=2 \pi i / \alpha$, so that the $H_{j}^{*}$ each have period $\tau_{3}(j=1,2)$. From (37), we obtain

$$
H_{j}^{*}\left(z+\tau_{j}\right)-H_{j}^{*}(z)=\left(\exp \left(a_{j} \tau_{j}\right)-1\right) \exp \left(a_{j} z+G_{j}(z)\right) .
$$

Consequently, $\exp \left(a_{j} z+G_{j}(z)\right)$ is periodic with period $\tau_{3}(j=1,2)$. Since $\tau_{j} / \tau_{3}$ $(j=1,2)$ cannot both be rational, it follows by applying Lemma 6 to

$$
\exp \left(a_{j}\left(z+\tau_{3}\right)+G_{j}\left(z+\tau_{3}\right)\right)=\exp \left(a_{j} z+G_{j}(z)\right) \quad(j=1,2),
$$


that either $G_{1}$ or $G_{2}$ must be doubly periodic and hence constant. Thus, (38) implies that one of the $a_{j} \tau_{j} / 2 \pi i$ is rational, contrary to our hypotheses. This completes the proof of Theorem 6 .

ReMARK. Note that when it is assumed that $G_{j}(z)$ are both nonconstant $(j=1,2)$, it then suffices to assume that $\exp \left(a_{j} \tau_{j}\right) \neq 1(j=1,2)$. This is clear from the proof.

As an immediate consequence of Theorem 6, we have

COROLlARY. Let $I_{1}$ and $I_{2}$ be complex numbers whose ratio $I_{1} / I_{2}$ is irrational, then the function

$$
F(z)=\exp \left(\left(2 \pi i / I_{1}\right) z\right)+\exp \left(\left(2 \pi i / I_{2}\right) z\right)
$$

is prime.

For in Theorem 6, we can write

$$
\begin{array}{ll}
H_{1}=\exp \left(\left(2 \pi i / I_{1}\right) z\right), & a_{1} z+G_{1}=\left(2 \pi i / I_{2}\right) z+0, \\
H_{2}=\exp \left(\left(2 \pi i / I_{2}\right) z\right), & a_{2} z+G_{2}=\left(2 \pi i / I_{1}\right) z+0,
\end{array}
$$

i.e. $\tau_{1}=I_{1}, \tau_{2}=I_{2}, a_{1}=2 \pi i / I_{2}, a_{2}=2 \pi i / I_{1}$. Then $F=F_{1}(z)=F_{2}(z)$ and any nontrivial right factor of $F$ would be a nontrivial common right factor of $F_{1}$ and $F_{2}$.

Thus, we have exhibited an almost periodic entire function which is prime.

We now prove a generalization of the above corollary.

THEOREM 7. For any nonconstant entire periodic functions $H(z)$ and $G(z)$ of period $\tau$ and any constant $\lambda$ such that $\lambda \tau / \pi i$ is irrational, the function

$$
F(z)=H(z)+e^{\lambda z+G(z)}
$$

is prime.

Proof. We assume again that $\tau=1$. Suppose that

$$
F(z)=f(g(z)),
$$

where $f$ and $g$ are entire and nonlinear. We conclude in the same manner as in the previous theorem that $g(z)$ has the form

$$
g(z)=H_{1}(z)+\exp \left(H_{2}(z)+c z\right),
$$

where $H_{j}$ is periodic with period $1(j=1,2)$.

(40) and (41) yield

$$
f\left(H_{1}(z)+e^{c n} \exp \left(H_{2}(z)+c z\right)\right)=H(z)+e^{\lambda z+G(z)} e^{\lambda n} .
$$

As in the previous theorem, we may assume that $\left|e^{c}\right|=1$. If $c$ is a rational multiple of $\pi i$, then for a fixed $z$ the left side of (42) is one of finitely many values while the right side runs through infinitely many values as $n$ runs through the integers. Thus, $e^{c n}$ for $n=1,2, \ldots$ is dense on the unit circle and we conclude as in the previous 
theorem that $f(w)$ must have the form

$$
f(w)=A(w-B)^{k}+C,
$$

where $A, B$ and $C$ are constants and $k$ is a positive integer.

Letting $H_{1}-B=H^{*},(41),(42)$ and (43) yield

$$
A\left(H_{1}^{*}+e^{c} \exp \left(H_{2}+c z\right)\right)^{k}-A\left(H_{1}^{*}+\exp \left(H_{2}+c z\right)\right)^{k}=\left(e^{\lambda}-1\right) e^{\lambda z+G(z)} .
$$

Expanding (44) we get an equation of the form

$$
T_{0}+T_{1} e^{c z}+\cdots+T_{k} e^{c k z}=e^{\lambda z},
$$

where $T_{i}$ are periodic with period 1 and not identically zero.

From (45) we get

$$
\left(e^{\lambda}-1\right) e^{\lambda z}=\left[T_{1}\left(e^{c}-1\right)+T_{2}\left(e^{2 c}-1\right) e^{c z}+\cdots+T_{k}\left(e^{k c}-1\right) e^{(k-1) c z}\right] e^{c z}
$$

which we can write in the form

$$
e^{(\lambda-c) z}=T_{1}^{1}+T_{1}^{1} e^{c z}+\cdots+T_{k-1}^{1} e^{(k-1) c z},
$$

where $T_{j}^{1}$ are periodic with period 1 and not identically zero. Continuing inductively, we get

$$
e^{(\lambda-(k-1) c) z}=T_{0}^{k-1}+T_{1}^{k-1} e^{c z}
$$

and finally

$$
e^{(\lambda-k c) z}=T_{0}^{k} .
$$

Clearly, $e^{(\lambda-k c) z}$ has period 1. Dividing both sides of (48) by $e^{c z}$ yields

$$
e^{(\lambda-k c) z}=T_{0}^{k-1} e^{-c z}+T_{1}^{k-1} .
$$

From (48) and (49) we conclude that $e^{c z}$ has period 1, a contradiction. This completes the proof of Theorem 7 .

V. Primes of every order. As we have noted earlier, Theorems 5 and 7 give examples of primes of arbitrarily large growth. It is also known (see [11, Theorem 5]) that any entire function of the form

$$
F(z)=H(z)+z^{2},
$$

where $H(z)$ is periodic and of finite lower order, has only quadratic right factors. Thus, for any even periodic $H(z)$ of order greater than or equal to 1 , the function $F(\sqrt{ } z)$ is prime and of order $\geqq \frac{1}{2}$. In fact it follows that for any $\rho$ and $\sigma$ with $\rho \geqq \frac{1}{2}$ and $\sigma \geqq 0$ (but $\neq 0$ when $\rho=\frac{1}{2}$ ) there exists a prime function of order $\rho$ and type $\sigma$. Of course we also have primes of order zero, namely prime polynomials.

We now consider a class of functions which include functions of arbitrarily small growth. We prove 
THEOREM 8. Let $\varphi(z)$ be any entire function of lower order less than 1 or of lower order equal to 1 and lower type equal to 0 . Let $n_{k}(k=1,2, \ldots)$ be any infinite sequence of positive integers such that at most finitely many of the $n_{k}$ are equal. Let $a_{k}$ be any sequence of positive reals such that $\prod_{k=1}^{\infty}\left(1-z / a_{k}\right)^{n_{k}}$ is of order less than 1 . If $\varphi(0)$ $\neq \varphi(1)$, then

$$
F(z)=e^{\varphi} z(z-1) \prod_{k=1}^{\infty}\left(1-\frac{z}{a_{k}}\right)^{n_{k}}
$$

is prime. The assertion remains valid when $\varphi$ is constant.

Before proceeding with the proof we shall need the following two results:

LEMMA 11 [12]. Let $b_{i}(i=1,2, \ldots, n)$ be any $n$ complex numbers. If $g$ is entire and if all but finitely many of the zeros of $g-b_{i}$ have multiplicity at least $m_{i}$ $(i=1,2, \ldots, n)$, then $\sum_{i=1}^{n}\left(1-1 / m_{i}\right) \leqq 1$.

LEMma 12 [18]. Let $f(z)$ be an entire function. Assume that there exists an unbounded sequence $\left\{h_{v}\right\}_{v=1}^{\infty}$ such that all the roots of the equations

$$
f(z)=h_{v} \quad(v=1,2,3, \ldots),
$$

are real. Then $f(z)$ is a polynomial of degree not greater than two.

Proof of theorem. Suppose that $F$ is not prime. By virtue of Lemma 4, we may write $F(z)=f(g(z))$, where $f$ and $g$ are both nonlinear and entire. It follows from Lemma 12 that either $f$ has at most finitely many zeros or $g(z)$ is a quadratic polynomial. Since the $a_{i}$ are positive, the latter is impossible. Thus, we may assume that $f$ is of the form $f(w)=Q(w) e^{\alpha(w)}$, where $Q$ is a polynomial and $\alpha$ is entire. If $Q(w)$ has two distinct zeros $b_{1}$ and $b_{2}$, say, then all but finitely many of the zeros of $g-b_{i}(i=1,2)$ have multiplicity greater than 3 . This is impossible by Lemma 11 . Since $z=0$ is a simple zero of $F$, it follows that $Q$ is linear. Hence, we may write $F(z)=g(z) e^{\alpha(g(z))}$, where $\alpha$ is entire. Clearly, $g$ has the form

$$
e^{\beta} z(z-1) \prod_{k=1}^{\infty}\left(1-\frac{z}{a_{k}}\right)^{n_{k}},
$$

where $\beta$ is entire. Since

$$
\alpha(g(z))=\varphi(z)-\beta(z)+T,
$$

$T$ a constant, and since $\varphi$ is at most of lower order 1 and lower type 0 , it follows by Lemma 1 , that either $\alpha$ or $\beta$ is a constant. When $\beta$ is a constant, we have from (51) that $\varphi(0)=\alpha(0)+S=\varphi(1)$ for some constant $S$, contrary to our hypotheses. Thus, $\alpha$ must be constant and our proof is complete.

Note that the proof for the case when $\varphi$ is constant is included in the above. The author would like to thank J. Miles for some constructive suggestions with regard to the above proof. 
In a subsequent paper the author will discuss a number of extensions of this result. As an immediate consequence of Theorem 8 and the remarks preceding it, we have

COROLlaRY. There exist primes having prescribed order and type.

VI. Some open questions. An argument similar to the one used in the proof of Theorem 2 can be used to prove

THEOREM 9. Let $H(z)$ be an entire, periodic function with period $\tau$. If $H(z)$ is of finite lower order, then for any nonzero constant $a$, the function

$$
F(z)=H(z)+a z e^{(2 \pi i / \tau) z}
$$

is prime.

More generally it is reasonable to conjecture

CONJECTURE 1. If az in Theorem 9 is replaced by an arbitrary nonconstant polynomial which is not of the form $P^{n}+C$, where $n$ is an integer $>1, P$ is a polynomial and $C$ is $Q$ constant, then the resulting function $F(z)$ is still prime.

It is shown in [1, Theorem 4] that when $H$ is periodic of exponential type, then any entire function of the form

$$
F(z)=H(z)+Q(z),
$$

where $Q(z)$ is a nonconstant polynomial, can only have factorizations of the form (14). It follows, in particular, that when $Q(z)$ in (52) is of odd degree, then $F$ is prime. This together with (50) suggests the following extension of Theorem 2.

CONJECTURE 2. For any periodic entire function $H(z)$ of finite lower order and any polynomial $Q(z)$ which has no quadratic right factor, $F$ in $(52)$ is prime.

We conclude this study by suggesting a number of additional functions which seem to be good candidates for being prime.

(1) Any function of the form $Q(z) e^{\alpha(z)}+P(z)$, where $Q(z)$ and $P(z)$ ( $\not$ constant) are polynomials with no common right factor. When $Q$ is constant we also assume that $\alpha$ and $P$ have no common right factor.

(2) All functions $z^{k+1 / 2} \sin \sqrt{ } z(k= \pm 1, \pm 2, \ldots)$.

(3) All functions $z^{i} \cos \sqrt{ } z(i= \pm 1, \pm 2, \ldots)$.

(2) and (3) suggest looking at the factorizations of functions of the form $P(z) H(z)$, where $H(z)$ is periodic of exponential type and $P(z)$ is a nonconstant polynomial.

\section{REFERENCES}

1. I. N. Baker and F. Gross, Further results on factorization of entire functions, Proc. Sympos. Pure Math., vol. 11, Amer. Math. Soc., Providence, R. I., 1968, pp. 30-35. MR 38 \#6066.

2. $\longrightarrow$, On factorizing entire functions, Proc. London Math. Soc. (3) 18 (1968), 69-76. MR 36 \#5344.

3. W. H. J. Fuchs and F. Gross, Generalization of a theorem of A. and C. Rényi on periodic functions, Acta Sci. Math. (Szeged) (to appear). 
4. F. Gross, On factorization of meromorphic functions, Trans. Amer. Math. Soc. 131 (1968), 215-222. MR 36 \#3988.

5. - The periodicity of compositions of entire functions, Canad. J. Math. 18 (1966), 724-730.

6. - On factorization of elliptic functions, Canad. J. Math. 20 (1968), 486-494. MR 37 \#1612.

7. - On the periodicity of $f(g)$, Amer. Math. Monthly 75 (1968), 279-280. MR 37 \#2989.

8. - On the periodicity of compositions of entire functions. II, Canad. J. Math. 20 (1968), 1265-1268. MR 38 \#1259.

9. - Some theorems on factorization of meromorphic functions, Bull. Amer. Math. Soc. 74 (1968), 649-650. MR 37 \#1614.

10. - On the growth of $f(g)$, Bull. Amer. Math. Soc. 74 (1968), 1030-1033. MR 39 \#7108.

11. - Factorization of entire functions which are periodic mod $g$, Proc. Nat. Inst. Sci. India Part A (to appear).

12. W. K. Hayman, Meromorphic functions, Oxford Math. Monographs, Clarendon Press, Oxford, 1964, p. 47. MR 29 \#1337.

13. R. Nevanlinna, Théorème de Picard-Borel et la théorie des fonctions meromorphes, Gauthier-Villars, Paris, 1929, pp. 112-121.

14. M. Ozawa, On the solution of the functional equation $f(g(z))=F(z)$. I, II, Kōdai Math. Sem. Rep. 20 (1968), 159-169. MR 37 \#4260.

15. G. Pólya, On an integral function of an integral function, J. London Math. Soc. 1 (1926), $12-15$.

16. A. Rényi and C. Rényi, Some remarks on periodic entire functions, J. Analyse Math. 14 (1965), 303-310. MR 31 \#2406.

17. P. C. Rosenbloom, The fix-points of entire functions, Medd. Lunds Univ. Mat. Sem. 1952, 186-192 (tome supplémentaire). MR 14, 546.

18. A. Edrei, Meromorphic functions with three radially distributed values, Trans. Amer. Math. Soc. 78 (1955), 276-293. MR 16, 808.

U.S. Naval Research Laboratory, Washington, D.C. 20390

UNIVERSITY OF MARYLAND, BaLTIMORE, MARYLAND 21228 